already mentioned. The program director or an assistant professor was asked to fill the survey out between April 2019 and January 2020. The sample was described through means and standard deviations or frequency and percentages according to the type of variable.

Results: Data was obtained from all the programs of specialization in internal medicine $(n=29)$, family medicine $(n=9)$, pain medicine $(n=8)$, physical and rehabilitation medicine $(n=6)$, geriatrics $(n=4)$. All the programs of internal medicine, geriatrics, and physical/rehabilitation medicine have rheumatology as a mandatory subject within the curriculum, while in family medicine and pain medicine programs: $33 \%(n=3)$, and $57 \%$ $(n=4)$ respectively, does not have a rheumatology training. In internal medicine the average duration of rheumatology training is 56 days (28 to 90 days), in geriatrics, it is 56 days (45-60 days), in family medicine 26.1 days ( 3 to 30 ), in medicine physical and rehabilitation medicine 35 days ( 30 to 60 ) and in pain medicine 40 days ( 30 to 60 ). $93.8 \%(n=45)$ of the programs surveyed have a rheumatologist as a teacher in charge of both, the academic activities and the external rheumatology consultation and in the rest of the programs these activities are done by an specialist in internal medicine, family medicine or pain medicine. The theoretical components most frequently offered in seminars or master classes are: rheumatoid arthritis, lupus, vasculitis, spondyloarthritis, gout, osteoarthritis, sjogren's syndrome and osteoporosis. The specialization programs have an average of 3 rheumatologists as professors (range 1 to 8 ). $43.8 \%$ of the programs use some virtual resources to complement the teaching in rheumatology such as video conferencing in virtual platform or YouTube (8.3\%), online course $(6.3 \%)$, mobile application (16.7\%), others such as moodle and webcast platform (12.5\%). 92.9\% (n = 52) offer their students an elective rotation in the rheumatology service, in addition to the mandatory one. Finally, $89.6 \%(n=43)$ of the interviewees agree with the way in which the curriculum is structured in their programs, to approach initial diagnostic and therapeutic in patients with suspected or established rheumatic disease. However, $23.2 \%(n=13)$ of the professors of the surveyed programs ( 6 of internal medicine, 4 of family medicine, 2 of pain medicine and 1 of physical/rehabilitation medicine), consider that the rheumatology subject should not be mandatory within their programs.

Conclusion: All physicians need to be familiar with rheumatological conditions since the rate of rheumatologists in Colombia is low. Knowing rheumatology is an increasingly important role in a clinical scenario in many postgraduate curriculums as we explain in this study.

References:

[1] Sivera F, et al; Working Group on Training in Rheumatology across Europe. Differences and similarities in rheumatology specialty training programmes across European countries. Ann Rheum Dis. 2015 Jun;74(6):1183-7

Disclosure of Interests: None declared

DOI: 10.1136/annrheumdis-2020-eular.6490

\section{AB1355-HPR THE IMPACT OF A STRUCTURED TRAINING PROGRAMME FOR THE DETECTION OF SYNOVITIS WITH MUSCULOSKELETAL ULTRASOUND (MSUS) IN RHEUMATOLOGY NURSING}

L. Cano Garcia' ${ }^{1}$, C. Dominguez ${ }^{2}$, S. Garcia Diaz ${ }^{3}$, R. Segura ${ }^{4}$, J. M. Martín Martín ${ }^{5}$ F. G. Jiménez-Núñez ${ }^{1}$ on behalf of OPEN REUMA. Instituto de Investigación Biomédica de Málaga (IBIMA), Hospital Regional Universitario de Málaga, Málaga, Spain; ${ }^{2}$ Hospital Virgen Macarena, Sevilla, Spain $;{ }^{3}$ Hospital Moisés Broggi, Sant Joan Despi, CSI, Barcelona, Spain; ${ }^{4}$ Hospital Reina Sofía, Córdoba, Spain; ${ }^{5}$ Hospital Universitario Nuestra Señora de la Candelaria, Tenerife, Spain

\section{Background:}

Objectives: To analyse the effect of a teaching intervention for the technical performance of a reduced index of ultrasound synovitis by nurses without previous experience in musculoskeletal ultrasound (MSUS)

Methods: Design: Quasi-experimental study of the before-after type. Protocol: The nurses received a theoretical-practical, face-to-face and intensive training of 8 hours, by an expert rheumatologist in musculoskeletal ultrasound (MSUS), to perform the Perricone index, which assesses the presence of grey-scale synovitis (GS) and quantifies the presence of a power-doppler (PD) signal semiquantitatively (0-3), in 6 joints (Wrists, 2nd Metacarpophalangeal (MCP) joints and knees). The theoretical knowledge acquired was assessed by an exam test type at the end of the training, and the technical skills were assessed by an ultrasound examination in patients with rheumatoid arthritis, immediately after the teaching session and at 18 months later. Variables: Illumination of the consultation, protection of privacy and unequivocal identification of the patient, technique (explorer ergonomics, bilateral comparison, use of both hands, measurement, marking and saving data), GS (probe position, centred image, cortical-cartilage-tendon display, depth, focus, frequency, gain), PD (probe pressure, PRF, position-width-depth of the box, focus, frequency, gain), synovial recesses (dorsal and palmar examination of the wrist, 2nd Metacarpophalangeal (MCP) joints and supra and parapatellar medial and lateral examination of the knees) and semi-quantitative gradation of synovitis in GS and PD. Statistical analysis: descriptive analysis, and in relation to the statistical significance tests for paired variables (pre and post teaching intervention) the McNemar test was used for dichotomous qualitative variables and McNemar-Bowker test for those of more than two categories.

Results: 5 nurses, $80 \%$ women, aged between 36 and 54 years participated. They adequately answered $100 \%$ of the 4 -student test questions, and the remaining one matched $80 \%$ of them. The average time of baseline exploration was $45,2 \pm 3,8$ minutes and the final time was $32,6 \pm 3,5$, improving the 5 students in an average of $12,6 \pm 4,4$ minutes. The technical aspects not performed correctly in the baseline ultrasound examination were the bilateral comparative, centred image, grey scale gain, measuring, correct anatomical image of the synovial recess of the wrist and position-width-depth of the Doppler box. An improvement was observed at 18 months in the unequivocal identification of the patient, adequate illumination of the consultation, bilateral comparison, correct anatomica acquisition of synovial recesses and the quantification of GS synovitis, but no statistically significant differences were observed, before and after the teaching intervention, in possible relation with the difficulty to use the ultrasound in their respective Rheumatology Units in clinical practice, but mainly because the aspects correctly performed in the baseline exploration were numerous.

Conclusion: A formal training of rheumatology nurses in musculoskeletal ultrasound (MSUS) could be very useful, and cost-efficient, in the health care of patients with rheumatoid arthritis

Disclosure of Interests: None declared

DOI: 10.1136/annrheumdis-2020-eular.4732

\section{AB1356-HPR GOUT IN SPANISH PRIMARY HEALHCARE CENTERS:} STILL A LONG WAY TO GO

M. A. Pou ${ }^{1}$, S. Jeria ${ }^{2}$, A. Prada-Ojeda ${ }^{3}$, S. Ferrer ${ }^{1}$, A. García-Guillén ${ }^{2}$ H. Corominas ${ }^{2}$, C. Diaz Torne ${ }^{2} .{ }^{1}$ EAP Encants, Barcelona, Spain; ${ }^{2}$ Hospital de la Santa Creu i Sant Pau, Rheumatology, Barcelona, Spain: ${ }^{3}$ Hospital de Torrejon, Madrid, Spain

Background: Gout has a prevalence $>2.5 \%$ in the Spanish adult population. It is a chronic disease that without proper treatment causes pain, joint deformity and increased cardiovascular risk and mortality. Recent advances have demonstrated that if correctly treated the disease can be controlled and even 'cured.' Most gouty patients are diagnosed and treated by general practitioners (GPs). There is evidence that the management if these patients is not good neither at Rheumatology Units nor at Primary Healthcare (PHC) centers.Several causes of this mismanagement can be found in the literature.

Objectives: Design and evaluation of the results of a questionnaire created from a bibliographic search focused on areas of improvement of gout management in PHC. Methods: A search was made in Pubmed to identify the main barriers described in the management of patients with gout in primary care. The terms used were: "Gout" "primary healthcare" and "education." A Google Form of gout knowledge and management questionnaire was designed, taking into account what is described in the literature. The Google Form was sent to all GP from an urban area via mail and to other contacts via WhatsApp and twitter.

Results: Responses were obtained from 224 GPs; 69.5\% were women; $73.1 \%$ had between 11 and 30 years of professional experience; $96.4 \%$ answered that gouty are mostly controlled in primary care; $99.6 \%$ performs the diagnosis of gout without analysis of synovial fluid and $17 \%$ diagnosed only by clinics without urate levels; $55.9 \%$ of GPs do not use any reference guide. Of those who use, the $73 \%$ use GUIPCLINGOT and $40 \%$ use SEMGs one; $80.5 \%$ have not done any gout course in the last 5 years; $26 \%$ did not have access to a rheumatologist to confirm the gout diagnosis; only $30.8 \%$ knew the therapeutic objective of the urate lowering therapy (ULT); $28.6 \%$ considered the beginning of ULT after the first attack; $62 \%$ believed that the most important part of the treatment was changing diet and lifestyles; $88.8 \%$ did not perform any specific education for these patients by the nurse just $37.2 \%$ carried out a treat-to-target strategy to lower urate levels.

Conclusion: The questionnaire identifies multiple points of improvement for the management of this pathology in accordance with the described in the literature. Most GPs are unaware of the therapeutic objective of the ULT.

Disclosure of Interests: None declared

DOI: 10.1136/annrheumdis-2020-eular.3117

\section{AB1357-HPR DESIGNING THE ROYAL COLLEGE OF NURSING COMPETENCY FRAMEWORK FOR RHEUMATOLOGY NURSES}

P. Livermore ${ }^{1}$, D. Finney ${ }^{1}$, J. Begum ${ }^{1}$, R. Wyllie ${ }^{1}$, T. Cornell ${ }^{1}$, H. Smith ${ }^{1}$,

L. Howie $^{1}$, L. Parker ${ }^{1}$ on behalf of Royal College of Nursing Rheumatology Nurse Forum Competency working party. ${ }^{1}$ Royal College of Nursing, London, United Kingdom

Background: The 1st Edition of the RCN Competency Framework for Rheumatology Nurse Specialists (RNS) will be published in March 2020. The role of the RNS is complex, and can include caring for children and young people. The importance of RNS's was highlighted by the National Rheumatoid Arthritis Society (NRAS 2017). The British Society for Rheumatology (BSR) outlined the need for education, training supervision and work force development. Education for RNS isn't currently centralized but is key to improving skills and developing workforce for the future. RNS are in short supply resulting in problems of access to services and delays in care (BSR 2019). In all 4 UK nations the titles of RNS and proficiency vary greatly (Titrate trial 2019) This is likely to have an impact on patient experience and outcomes. The European League Against Rheumatism (EULAR) developed 